\section{Dor crônica em idosos residentes em São Paulo, Brasil: prevalência, características e associação com capacidade funcional e mobilidade (Estudo SABE)}

\author{
Chronic pain among elderly residents in São Paulo, \\ Brazil: prevalence, characteristics, and association \\ with functional capacity and mobility (SABE Study)
}

\author{
Dolor crónico en los ancianos que viven en São \\ Paulo, Brasil: prevalencia, características y \\ asociación con la capacidad funcional y \\ la movilidad (Estudio SABE)
}

Mara Solange Gomes Dellaroza 1 Cibele Andrucioli de Mattos Pimenta 2 Yeda Aparecida Duarte 2 Maria Lúcia Lebrão 3

\footnotetext{
${ }_{1}$ Departamento de Enfermagem, Universidade Estadual de Londrina, Londrina, Brasil.

2 Escola de Enfermagem, Universidade de São Paulo, São Paulo, Brasil.

3 Faculdade de Saúde Pública, Universidade de São Paulo, São Paulo, Brasil.



\begin{abstract}
The objective was to identify the prevalence and characteristics of chronic pain and the association with functional capacity. This was a cross-sectional study with a population sample of community-dwelling elderly residents in the city of São Paulo, Brazil, using home interviews. Statistical analysis used the RaoScott test of association with $p<0.05$. Prevalence of chronic pain was 29.7\% (95\%CI: 25.4-33.9), most frequent in the lower back (25.4\%) and lower limbs (21.9\%), and moderate in $45.8 \%$ and intense in $46 \%$ of the sample. Chronic pain was associated with increased dependence and decreased mobility $(p<0.001)$.
\end{abstract}

Chronic Pain; Low Back Pain; Health of the Elderly

\section{Resumo}

O objetivo foi identificar a prevalência, as características e a associação da dor crônica com capacidade funcional. Estudo transversal com amostra populacional feito por entrevista domiciliar com idosos residentes na cidade de São Paulo, Brasil. Dor crônica foi aquela com duração mínima de seis meses. Nas análises estatísticas utilizou-se teste de associação de RaoScott com significância de $p<0,05$. Dor crônica ocorreu em 29,7\% dos idosos (IC95\%: 25,4-33,9); os locais mais frequentes foram a região lombar $(25,4 \%) e$ os membros inferiores (21,9\%) e foi moderada em $45,8 \%$ das vezes e intensa em $46 \%$ dos idosos. Dor crônica esteve associada à maior dependência e pior mobilidade $(p<0,001)$.

Dor Crônica; Dor Lombar; Saúde do Idoso 


\section{Introdução}

O envelhecimento populacional é realidade no Brasil. Dados do Censo Demográfico de 2010 (Instituto Brasileiro de Geografia e Estatística. Sinopse dos resultados do censo 2010. http: / / www. censo2010.ibge.gov.br/sinopse/webservice/de fault.php? $\operatorname{cod} 1=35 \& \operatorname{cod} 2=355030 \& \operatorname{cod} 3=35 \& \mathrm{f}$ rm, acessado em 14/Fev/2012) demonstraram que a porcentagem de idosos atingiu $11,3 \%$ da população.

O processo de envelhecimento é acompanhado pela alta incidência de doenças crônicas e degenerativas 1,2 , muitas vezes com presença de dor crônica e elevada dependência $3,4,5$. Dor crônica é aquela associada a processos patológicos crônicos, que se prolongam por meses ou anos e, em muitos desses quadros, a dor é a principal queixa e causa de limitações funcionais. Estudos brasileiros observaram alta prevalência de dor crônica nos indivíduos acima de 60 anos residentes na comunidade (variando entre $51 \%$ e $67 \%$ ), especialmente dores musculoesqueléticas $(47 \%$ a $14 \%) 6,7,8,9$.

Embora a dor não apareça como fator direto de dependência e morte em muitos estudos, algumas pesquisas epidemiológicas comprovaram a interferência da dor em aspectos da vida e relacionaram-na com limitações funcionais $10,11,12,13,14,15$.

É ainda um desafio provar como e quais os mecanismos envolvidos na incapacidade provocada pela dor. Weiner et al. 16, em estudo caso controle com 323 idosos, comprovaram que a incapacidade provocada pela dor lombar em idosos residentes na comunidade é mediada por alterações neuropsicológicas. Entre as alterações detectadas, foi significativo o impacto na habilidade de atenção e na viso-espacial, avaliadas por uma bateria de testes. Estas descobertas ampliam as explicações dos mecanismos envolvidos na dor incapacitante e podem direcionar novas terapêuticas e pesquisas.

A incapacidade resultante da dor é multideterminada, assim, localizações álgicas diversas e intensidades diferentes, entre outras características dolorosas, parecem estar associadas ao maior ou menor grau de incapacidade.

No Brasil foram encontrados poucos estudos epidemiológicos sobre prevalência de dor crônica em idosos comunitários 6,13,17, todos com população de conveniência e nenhum em uma grande metrópole. Embora os resultados alertem para a magnitude do problema, pouco se sabe sobre o ônus social da dor crônica e de como esta interfere na qualidade de vida e na capacidade funcional dos idosos brasileiros. Assim, os objetivos deste estudo foram identificar a prevalên- cia e características da dor crônica de idosos da comunidade de uma grande metrópole e avaliar a associação de dor crônica com capacidade funcional e mobilidade.

\section{Método}

Trata-se de estudo epidemiológico transversal e populacional. Os dados foram provenientes do banco de dados do projeto $S A B E$ - Saúde, Bemestar e Envelhecimento. Este projeto é um estudo multicêntrico, coordenado pela Organização Pan-Americana da Saúde 18 e visa identificar as condições de vida e de saúde, e, avaliar as demandas de saúde de idosos residentes em sete cidades da América Latina e Caribe. A cidade escolhida no Brasil foi São Paulo. No Brasil, o SABE constitui-se em um estudo longitudinal, com três ondas, em 2000, 2006 e 2010, coordenado por uma equipe de pesquisadores da Faculdade de Saúde Pública, da Universidade de São Paulo (USP).

Para a realização de estudos sobre dor, em 2006, foram incluídas questões sobre dor crônica e, na presente pesquisa, serão analisados os dados coletados em 2006. Assim o presente estudo constitui-se em um estudo transversal que utilizou dados da coleta do ano de 2006 do estudo SABE que no Brasil constitui-se em um estudo longitudinal.

\section{População e amostra}

A amostra de 2006 foi constituída pelos indivíduos da onda de 2000 que sobreviveram, acrescidos de uma nova coorte de 298 idosos como explicitado a seguir. Dos 2.143 revisitados entrevistados em 2000, foi possível reentrevistar 1.115 idosos em 2006. As entrevistas não realizadas ocorreram devido a 649 óbitos, 178 recusas, 139 idosos não localizados, 51 mudanças para outros municípios e 11 institucionalizações. Para manter a mesma amplitude etária de 60 anos e mais, optou-se por realizar a inclusão de uma nova coorte de idosos entre 60 e 64 anos, com um número de 400 indivíduos. Ao final das buscas foram identificadas 376 pessoas e realizadas 298 entrevistas, devido a 72 recusas, 1 óbito e 5 mudanças de moradia. Assim, na coorte de 2006, 1.413 idosos foram entrevistados. Os pesos amostrais aplicados em 2000 foram recalculados, de forma a garantir a representatividade da amostra de 2006, relativamente à população de 60 anos ou mais do município. A técnica de amostragem do estudo SABE está detalhada em alguns textos 19,20.

Dessa forma, paralelamente à realização da pesquisa com a coorte $\mathrm{A}$, introduziu-se uma nova 
coorte de 60 a 64 anos. A população de estudo foi composta por pessoas residentes, no ano de 2006, na área urbana do Município de São Paulo. Em 2004, o seu tamanho era de 310.694 habitantes, o que correspondia a $2,9 \%$ da população, segundo as estimativas preliminares dos totais populacionais divulgadas pelo Instituto Brasileiro de Geografia e Estatística (IBGE; http:// www.ibge.gov.br/home/estatistica/populacao/ estimativa2008/default.shtm, acessado em 10/ Nov/2011).

A amostra foi composta por 400 indivíduos, o que define a fração de amostragem 400/310.694 $=0,0012874$. Para o cálculo do tamanho da amostra, considerou-se a prevalência de hipertensão igual a $50 \% 21$, o efeito de delineamento igual a 1,5 e margem de erro de $7 \%$. Correções para taxa esperada de resposta (75\%) e mortalidade igual a $2 \%$ foram introduzidas 18,19 .

Para o sorteio dos domicílios foi usado o método de amostragem por conglomerados, em dois estágios, sob o critério de partilha proporcional ao tamanho (PPT). As unidades de primeiro e segundo estágios foram, respectivamente, o setor censitário e o domicílio. Foram sorteados no primeiro estágio 40 setores, observando-se o critério segundo o qual o número de Unidades Primárias de Amostragem (UPAs) deveria ser $\geq 30$ e o número mínimo de entrevistas por setor igual a $400 / 40=10$.

O número mínimo de domicílios sorteados por setor foi de 118 , calculado pela expressão [d = $\left.\left(10^{*} 10 / 1\right)^{*}(0,85)^{-1}\right]$, cujos termos correspondem a 10 entrevistas por setor, a razão 10/1 é o inverso de 1 idoso para cada 10 domicílios e 0,85 é a taxa esperada de sucesso da operação de localização e realização das entrevistas nos domicílios particulares permanentes sorteados. Para o conjunto dos 40 setores foram sorteados 4.720 domicílios.

Ao final do processo foram identificadas 375 pessoas dentro do critério estabelecido (60 a 64 anos) e foram realizadas 298 entrevistas.

$O$ resultado final pode ser visto a seguir: (a) 6.964 domicílios sorteados; (b) 275 segmentos sorteados; (c) 375 idosos localizados pelas "batedoras”; (d) 7 perdas (6 mudanças e 1 óbito); (e) 70 recusas (11,55\%); (f) 298 antropometrias realizadas; (g) 298 exames bucais realizados e; (h) 298 entrevistas realizadas.

O questionário utilizado foi o mesmo do inquérito de 2006.

\section{Variáveis independentes}

Idosos com dor crônica foram considerados aqueles que referiram dor com duração igual ou superior a seis meses, conforme definição da International Association for the Study of Pain 22.
A dor crônica que mais incomodava os idosos foi caracterizada conforme sua localização, duração, intensidade, frequência (Tabela 1) e seu impacto em aspecto da vida. A intensidade da dor foi avaliada através de descritores verbais: dor fraca, moderada/média, forte/intensa e muito forte/muito intensa.

\section{Variáveis dependentes}

Na capacidade funcional avaliou-se a realização de atividades de vida diária e atividades instrumentais de vida diária. As questões sobre atividades básicas de vida diária incluíram: atravessar um quarto da casa, vestir-se, tomar banho, fazer higiene pessoal - lavar as mãos e o rosto, pentear-se, barbear-se - comer sozinho, deitarse e levantar da cama e cadeiras e usar o vaso sanitário. As atividades instrumentais de vida diária, investigadas por meio da escala de Lawton, foram: preparar uma refeição quente, cuidar de seu dinheiro, utilizar transporte, fazer compras, telefonar, fazer tarefas domésticas leves e mais pesadas e tomar remédios 23 .

Para cada atividade, foi questionado ao idoso se ele tinha dificuldade para fazê-las e as alternativas foram: sim, não, não consegue, não costuma fazer. Assim, os idosos foram classificados em independente: aqueles que responderam não ou não costumam fazer todas as atividades avaliadas e indivíduo dependente, aqueles que responderam sim ou não conseguem fazer, no mínimo, uma atividade avaliada.

A mobilidade foi avaliada por meio do autorrelato de dificuldade na realização de diversas ações e movimentos, entre elas caminhar uma rua (quadra); ficar sentado(a) durante duas horas, levantar-se de uma cadeira, curvar-se, ajoelhar-se ou agachar-se. As categorias utilizadas foram: mobilidade alterada, sim, se o idoso relatou dificuldade em uma das atividades, respondendo "sim" ou "não pode fazer"; e não: se o idoso não se referiu à dificuldade respondendo; "não" ou "pode mas não faz".

\section{Aspectos éticos}

O projeto SABE recebeu a aprovação do Comitê de Ética em Pesquisa da Faculdade de Saúde Pública da USP, conforme of. COEP/83/06 e, para o desenvolvimento deste estudo, foi obtida a autorização dos coordenadores do SABE.

\section{Análise estatística}

Os dados foram analisados pelo programa estatístico Stata 11.0 (Stata Corp., College Station, Estados Unidos). Foi realizada a estatística 
Características da dor crônica.

\begin{tabular}{|c|c|c|}
\hline Características & Questão & Categorias de análise \\
\hline \multirow[t]{3}{*}{ Local da dor } & Qual é o local da dor que mais o(a) incomoda? Assinale o & 1. Membros inferiores * \\
\hline & mais importante (apenas o local) & 2. Região lombar \\
\hline & & 3. Outras regiões \\
\hline \multirow[t]{3}{*}{ Duração da dor } & Há quanto tempo dura essa dor ou a dor que mais o(a) incomoda? & 1. 6 meses e menos de 1 ano \\
\hline & & 2. 1 a 2 anos \\
\hline & & 3. Mais de 2 anos \\
\hline \multirow[t]{2}{*}{ Intensidade da dor } & Essa dor [a que mais o(a) incomoda] é? & 1. Fraca/Média/Moderada \\
\hline & & 2. Forte/Intensa/Muito forte/Muito intensa \\
\hline \multirow[t]{4}{*}{ Frequência da dor } & Essa dor [a que mais o(a) incomoda] dói (em média)? & 1. Quase todos os dias \\
\hline & & 2. Uma ou duas vezes por semana \\
\hline & & 3. Uma vez a cada 15 dias \\
\hline & & 4. Uma vez por mês \\
\hline
\end{tabular}

* Inclui segmento de coxa, perna, pé e articulações.

descritiva das variáveis sociodemográficas e das características da dor crônica que mais incomodava os idosos. Para analisar a associação entre dor e capacidade funcional e mobilidade utilizou-se o teste de associação de RaoScott 24,25.

\section{Resultados}

\section{Casuística}

Foram incluídos os idosos que responderam aos questionários sozinhos $(\mathrm{n}=1.199)$ ou com ajuda de informante auxiliar durante a coleta de dados $(\mathrm{n}=72)$ totalizando 1.271 idosos. Dos 1.413 entrevistados no SABE 2006 foram excluídos 142 idosos impossibilitados de responder e cujas informações foram fornecidas por um informante substituto que não o próprio idoso. Foram considerados impossibilitados de responder idosos com alterações de fala e audição, intensa debilidade física e déficit cognitivo. Os idosos com déficit cognitivo foram todos os idosos com pontuação no Mini Exame de Estado Mental (MEEM) menor que 13 pontos e menos que 6 na escala Pfeffer Functional Activities Questionnaire (QPAF).

Conforme o desenho amostral, os 1.271 idosos incluídos correspondem a 969.560 idosos residentes na cidade de São Paulo, ou seja, 99,7\% da população de idosos em 2000 , e $72,4 \%$ da população de 1.339.138 idoso, segundo o Censo Demográfico de 2010 (Tabela 2).

Dentre os idosos entrevistados, a prevalência de dor crônica foi de 29,7\% (intervalo de $95 \%$ de confiança - IC95\%: 25,4-33,9) representando 287.329 idosos.
Na Tabela 3, estão apresentados os dados sobre as características da dor crônica que mais incomodava os idosos e o impacto em aspectos do dia a dia.

Para a maioria, a dor que mais incomodava persistia há mais de dois anos $(77,4 \%)$ e variava, em intensidade, de média à intensa $(73,3 \%)$.

A presença da dor crônica mostrou-se associada à pior capacidade funcional avaliada por meio das atividades de vida diária básica e instrumentais e mobilidade (Tabela 4).

\section{Discussão}

A caracterização apresentada coincide com as condições da população idosa das últimas pesquisas nacionais. $\mathrm{O}$ quadro álgico apresentado pelos idosos desta pesquisa demonstra a importância da queixa de dor e o nível de sofrimento que ela impõe a esses indivíduos, o que coincide com achados em todo o mundo, inclusive no Brasil.

Poucos são os estudos epidemiológicos brasileiros sobre dor crônica em idosos residentes na comunidade. Na análise de um inquérito de saúde do Estado de São Paulo, dados dos indivíduos com mais de 60 anos apontaram prevalência de $30 \%$ de dor lombar, sem detalhamento das características dessa dor 8 . Estudo com idosos residentes na comunidade também em São Paulo, com amostra de 361 indivíduos, encontrou prevalência de $5,5 \%$ de fibromialgia, $14,1 \%$ de dor generalizada, $54,6 \%$ de dor regional ou em um segmento do corpo e somente $27,7 \%$ não referiram dor alguma ${ }^{9}$. 
Distribuição de idosos segundo características sociodemográficas. São Paulo, Brasil, 2006 *.

\begin{tabular}{lc}
\hline Características & $\%$ \\
\hline Faixa etária (anos) & 29,7 \\
$60-64$ & 48,4 \\
$65-74$ & 21,9 \\
$\geq 75$ & $69,5(0,6)$ \\
$\quad$ Média (erro-padrão) & \\
Sexo & 59,6 \\
$\quad$ Feminino & 40,4 \\
Masculino & \\
Trabalha & 68,3 \\
Não & 31,7 \\
Sim & \\
Escolaridade (anos) & 15,2 \\
0 & 59,4 \\
$1-4$ & 12,0 \\
$5-8$ & 13,4 \\
$>8$ & $4,3(0,2)$ \\
Média (erro-padrão) & \\
Vive sozinho & 86,6 \\
Não & 13,4 \\
Sim & \\
Renda mensal do idoso (salários mínimos) ** & 25,0 \\
$\leq 1$ & 42,8 \\
$>1$ e > 3 & 32,2 \\
$\geq 3$ & \\
\hline &
\end{tabular}

* $N=1.271$ representando 969.560 pessoas idosas.

** Salário mínimo: R\$350,00 - valor de referência no ano de 2006, que corresponde a US\$148,93 (valor do US\$ em janeiro de $2006=\mathrm{R} \$ 2,35$.

Em inquérito domiciliar com 451 idosos da comunidade da cidade de Londrina (Paraná), observou-se dor com duração de 6 meses ou mais em 51,44\% dos avaliados 6 , valor superior ao observado na presente pesquisa $(29,7 \%)$. Os locais mais frequentes de dor crônica foram região dorsal $(21,7 \%)$ e membros inferiores $(21,5 \%)$, à semelhança do observado na presente pesquisa (idosos da cidade de São Paulo) e ao descrito na literatura 7 . No estudo de Londrina, 38,4\% relataram dor moderada e $10 \%$, dor intensa, totalizando cerca de $50 \%$ de queixa de dor moderada ou intensa, percentagem inferior à observada na presente pesquisa que observou dor de moderada a intensa em cerca de $90 \%$ dos casos. Além disso, 32,6\% das dores foram diárias e, na presente pesquisa, dor diária ocorreu em quase 50\% dos investigados. É possível que essa diferença deva-se à população investigada, pois, no estudo atual a amostra representa idosos da cidade de São Paulo e no estudo de Londrina, a amostra referia-se aos idosos funcionários públicos da prefeitura da cidade de Londrina, portanto, muito mais restrita.

Von Korff et al. 26 , ao pesquisarem a dor na coluna que ocorreu nos 12 meses antes da entrevista, encontraram entre os acima de 60 anos ( $\mathrm{n}=971$ ), prevalência de dor durante "a vida" de $36,6 \%$ e de $21,6 \%$ nos últimos 12 meses. Os autores não informaram o critério para definição de dor crônica quanto à duração da dor. Este valor se assemelha a prevalência de $29,7 \%$ do presente estudo.

Considerando-se diversos estudos, nota-se que as prevalências variam, para dores em geral de $33 \% 25$ a $87,2 \% 28$, dependendo dos critérios para definição da dor crônica e amostra investigada. Para dor lombar, a variação dos estudos vai de $21,6 \%$ a $36,6 \% 26,29,30$; membros inferiores, os valores observados são de $18,4 \%$ a $27,8 \%$ 6,26,28, para partes especificas dos membros inferiores as variações são: quadril 11,6\% a 18,9\% 31,32, joelhos $14,7 \%$ a $18,9 \%$ 30,32 e pés e tornozelos de $12,1 \% 31$. 
Tabela 3

Características da dor crônica que mais incomoda os idosos. São Paulo, Brasil, 2006 *.

\begin{tabular}{lc}
\hline Características da dor que mais incomoda & $\%$ \\
\hline Tempo de dor & \\
6 meses a 11 meses & 9,2 \\
1 a 2 anos & 13,4 \\
Mais de dois anos & 77,4 \\
Local de dor & \\
Região lombar (abaixo da cintura) & 25,4 \\
Membros inferiores & 21,9 \\
Membros superiores & 13,0 \\
Região dorsal (acima da cintura) & 10,7 \\
Região cefálica & 10,5 \\
Região abdominal & 6,4 \\
Região cervical & 5,5 \\
Região torácica & 2,7 \\
Região pélvica & 2,4 \\
Região perineal e genital & 1,5 \\
Intensidade & \\
Fraca & 8,2 \\
Média/Moderada & 15,7 \\
Forte/Intensa & 1,5 \\
Muito forte/Muito intensa & 27,5 \\
Frequência dos episódios & 18,5 \\
Uma ou duas vezes por semana todos os dias & \\
Uma vez a cada 15 dias & 49,6 \\
Não sabe & 22,7 \\
\hline
\end{tabular}

* $n=377$ representando 287.329 pessoas idosas.

Tabela 4

Distribuição do grau de dependência e alteração da mobilidade dos idosos, segundo ocorrência da dor crônica. São Paulo, Brasil, 2006.

\begin{tabular}{|c|c|c|c|}
\hline \multirow[t]{2}{*}{ Variáveis } & \multicolumn{2}{|c|}{ Dor há 6 meses ou mais } & \multirow[t]{2}{*}{ Valor de $p$ * } \\
\hline & Não (\%) & $\operatorname{Sim}(\%)$ & \\
\hline Dependência nas atividades básicas da vida diária & & & $<0,001$ \\
\hline Idosos independentes & 89,0 & 66,3 & \\
\hline Idosos dependentes & 11,0 & 33,7 & \\
\hline Dependência nas atividades instrumentais da vida diária & & & $<0,001$ \\
\hline Idosos independentes & 57,4 & 37,6 & \\
\hline Idosos dependentes & 42,6 & 62,4 & \\
\hline Mobilidade alterada & & & $<0,001$ \\
\hline Não & 43,5 & 27,2 & \\
\hline Sim & 56,5 & 72,8 & \\
\hline
\end{tabular}

* Teste de RaoScott. 
Diferentes delineamentos de pesquisa e critérios variados para definição de dor crônica dificultam a comparação dos resultados. Prevalências mais elevadas são encontradas quando se utilizam como população de pesquisa idosos institucionalizados ou vinculados aos serviços de saúde. Esta diferença parece justificar-se, pois tais idosos apresentam comorbidades importantes. Estudos específicos sobre o tema dor também tendem a encontrar prevalências mais elevadas do que em inquéritos sobre saúde na qual a dor é uma das variáveis de análise, como ocorreu no estudo SABE 2006. Para a plena compreensão dessas variações mais estudos são necessários 10 .

Na presente pesquisa, a importância da queixa álgica que mais incomodava os idosos demonstrou-se pela associação dessa queixa ao maior grau de dependência nas atividades de vida diária básica e instrumental e na alteração da mobilidade.

Diferentes desfechos são utilizados para avaliar a incapacidade advinda da dor e os mais referidos são menor capacidade física para caminhar, subir escadas, levantar da cadeira, manter o equilíbrio e as atividades instrumentais da vida diária 8,9,10,17,28,31,33,34,35,36,37,38,39. A diversidade de instrumentos para avaliar a dor e a incapacidade provocada por ela limita a comparação entre os estudos.

Em estudo com americanos da comunidade, com 50 ou mais anos, para todas as variáveis de funcionalidade e mobilidade avaliadas, os idosos com dor apresentaram frequência maior de limitações. Outro achado relevante foi que indivíduos de 50 a 59 anos com dor tiveram desempenho similar aos de 80 a 89 anos sem dor 38 .

Algumas características da dor estão associadas a maiores déficit funcionais e incapacidade, como dores intensas 16,26,35,39,40, múltiplos locais de dor 28,36,39, dores em região dorsal 8,17,29,30,34 e em membros inferiores 17,34,39.

Pela análise dos estudos encontrados nota-se que a interferência da dor na capacidade funcional e mobilidade de idosos é fato comprovado mundialmente 8,9,10,17,28,31,33,34,35,36,37,38,39. Nossos resultados comprovaram, pela primeira vez, em um estudo populacional brasileiro, que a dor de idosos da comunidade é um agravo importante e que provoca incapacidades também em nosso meio.

Uma das fortalezas do presente estudo é a técnica de amostragem randomizada com capacidade de representativa dos idosos residentes na área urbana de São Paulo capital, possibilitando o aprofundamento da relação entre ocorrência de dor e esta população. A inclusão do tema dor na coleta SABE 2006, viabilizou a análise da prevalência e das consequências da dor crônica em idosos de uma metrópole brasileira, ampliando o entendimento deste agravo na vida dos idosos brasileiros.

Uma limitação é o fato de que os dados sobre capacidade funcional e mobilidade basearamse exclusivamente no autorrelato pelos idosos. O estudo não previu a aplicação de testes físicos que confirmassem as incapacidades referidas. Um fator que influência o autorrelato são as condições de saúde e ambientais das demais pessoas da comunidade onde o indivíduo reside. Sabe-se que a autopercepção sobre capacidade funcional sofre influência de fatores sociais e psicoemocionais do idoso.

Entretanto, foi realizada avaliação do estado cognitivo do idoso, o que assegurou maior confiabilidade das informações coletadas, mas limita a generalização destes resultados para idosos demenciados ou com alterações cognitivas.

Por tratar-se de uma análise transversal é possível demonstrar associação entre os fenômenos estudados, porém nenhuma relação de causalidade pode ser inferida.

Os resultados encontrados sobre interferência da dor em diferentes aspectos da vida demonstram que este é um agravo que precisa ser considerado na atenção ao idoso e no planejamento de ações que visem o bem-estar desta população. Ter a dor controlada é um direito da pessoa idosa, cada vez mais reconhecido por profissionais e esperado pela sociedade. O controle da dor ou a diminuição de sua intensidade podem ser estratégias para melhorar a capacidade funcional e a qualidade de vida de idosos portadores de dores crônicas. A gravidade da queixa álgica e sua interferência na qualidade de vida demonstrados em nossos achados alertam que o controle da dor precisa ser incluído como objetivo em programas de envelhecimento ativo.

\section{Conclusão}

A presente pesquisa, representativa da população idosa da maior cidade brasileira, observou prevalência de 29,7\% de dor crônica. Os locais mais frequentes da dor crônica que mais incomodava foram região lombar, membros inferiores e superiores. A dor crônica que mais incomodava foi diária para quase $50 \%$ dos idosos e possuía intensidade moderada para $45,8 \%$ deles e forte e muito forte para $46 \%$ dos idosos. A dor crônica que mais incomodava os idosos associou-se a incapacidades nas atividades de vida diária, nas atividades de vida instrumental e alteração de mobilidade. É preciso que a dor seja incluída na agenda de pesquisadores, gestores e profissionais que atuam na área de atenção ao idoso. 


\section{Resumen}

El objetivo fue identificar la prevalencia, las características y la asociación del dolor crónico con la capacidad funcional. Se trata de un estudio transversal con una muestra poblacional, realizada mediante entrevista domiciliaria con ancianos residentes en la ciudad de São Paulo, Brasil. Fue considerado dolor crónico aquel con una duración mínima de seis meses. En los análisis estadísticos se utilizó el test de asociación de Rao-Scott con significancia de $p<0,05$. El dolor crónico se produjo en un 29,7\% de los ancianos (IC95\%: 25,4-33,9); los lugares más frecuentes fueron la región lumbar $(25,4 \%)$ y los miembros inferiores $(21,9 \%)$ y fue moderado en un $45,8 \%$ de las veces e intenso en un $46 \%$ de los ancianos. El dolor crónico estuvo asociado a una mayor dependencia y peor movilidad $(p<0,001)$.

Dolor Crónico; Dolor de la Región Lumbar; Salud del Anciano

\section{Colaboradores}

M. S. G. Dellaroza foi responsável pela estruturação e elaboração do artigo na íntegra. C. A. M. Pimenta contribuiu diretamente na elaboração final do artigo em sua estrutura e conteúdo. Y. A. Duarte e M. L. Lebrão colaboraram na revisão do texto final.

\section{Agradecimentos}

O Estudo SABE obteve recursos da FAPESP. À Dra Patrícia E. Braga pela sua valiosa contribuição na realização das análises estatísticas e revisão dos resultados.

\section{Referências}

1. Almeida MF, Barata RB, Montero CV, Silva ZP. Prevalência de doenças crônicas auto referidas e utilização de serviços de saúde, PNAD/1998, Brasil. Ciênc Saúde Coletiva 2002; 7:743-6.

2. Instituto Brasileiro de Geografia e Estatística. Síntese de indicadores sociais: uma análise das condições de vida da população brasileira, 2010. Rio de Janeiro: Instituto Brasileiro de Geografia e Estatística; 2010.

3. Lima-Costa MF, Loyola Filho AI, Matos DL. Tendências nas condições de saúde e uso de serviços de saúde entre idosos brasileiros: um estudo baseado na Pesquisa Nacional por Amostra de Domicílios (1998-2003). Cad Saúde Pública 2007; 23: 2467-78.
4. Lima-Costa MF, Barreto S, Giatti L. Condições de saúde, capacidade funcional, uso de serviços de saúde e gastos com medicamentos da população idosa brasileira: um estudo descritivo baseado na Pesquisa Nacional por Amostra de Domicílios. Cad Saúde Pública 2003; 19:735-43.

5. Schmidt MI, Duncan BB, Silva GA, Menezes AM, Monteiro CA, Barreto SM, et al. Chronic non-communicable diseases in Brazil: burden and current challenges. Lancet 2011; 377:1949-61.

6. Dellaroza MSG, Pimenta CAM, Matsuo T. Prevalência e caracterização da dor crônica em idosos não institucionalizados. Cad Saúde Pública 2007; 23:1151-60. 
7. Panazzolo D, Trelha CS, Dellaroza MSG, Cabrera MAS, Souza R. Dor crônica em idosos moradores do Conjunto Cabo Frio, cidade de Londrina/PR. Rev Dor 2007; 8:1047-51.

8. Lima MG, Barros MBA, César CLG, Goldbaum M, Carandina L, Ciconelli RM. Impact of chronic disease on quality of life among the elderly in the state of São Paulo, Brazil: a population-based study. Rev Panam Salud Pública 2009; 25:314-21.

9. Santos AMB, Burti JS, Lopes JB, Scazufca M, Marques AP, Pereira RM. Prevalence of fibromyalgia and chronic widespread pain in communitydwelling elderly subjects living in São Paulo, Brazil. Maturitas 2010; 67:251-5.

10. Dellaroza MSG. Prevalência e caracterização da dor crônica em idosos servidores municipais de Londrina-PR [Dissertação de Mestrado]. São Paulo: Escola de Enfermagem, Universidade de São Paulo; 2000.

11. Lamb S, Guralnik J, Buchner D, Ferrucci L, Hochberg M, Simonsick E, et al. Factors that modify the association between knee pin and mobility limitation in older women: the women's health and aging study. Ann Rheum Dis 2000; 59:331-7.

12. Sokka T, Kankainen A, Hannonem P. Scores for functional disability in patients with rheumatoid arthritis are correlated at higher levels with pain scores than with radiographic scores. Arthritis Rheum 2000; 43:386-9.

13. Furuya RK, Dellaroza MSG, Cabrera MAS, Matsuo T, Trelha C, Yamada KN, et al. Caracterização da dor crônica e métodos analgésicos utilizados por idosos da comunidade. Rev Assoc Med Bras 2008; 54:36-41.

14. Camaciu C, Iliffe S, Kharicha K, Harari D, Swift C, Gillmann G, et al. Health risk appraisal in older people 3: prevalence, impact and context of pain and their implications for GPs. Br J Gen Practice 2007; 57:630-6.

15. Patil SK, Johnson AS, Lichtenberg PA. The relation of pain and depression with various healthpromoting behaviors in African-Americans elders. Rehabil Psychol 2008; 53:85-92.

16. Weiner DK, Rudy TE, Morrow L, Slaboda J, Lieber S. The relationship between pain, neuropsychological performance, and physical function in community-dwelling older adults with chronic low back pain. Pain Med 2006; 7:60-70.

17. Blay SL, Andreoli SB, Gastal FL. Chronic painful physical conditions, disturbed sleep and psychiatric morbidity: results from an elderly survey. Ann Clin Psychiatry 2007; 19:169-74.

18. Lebrão ML. O projeto SABE em São Paulo: uma visão panorâmica. In: Lebrão ML, Duarte YAO, organizadores. SABE - Saúde, Bem-estar e Envelhecimento. O projeto SABE no município de São Paulo: uma abordagem inicial. Brasília: Organização Pan-Americana da Saúde; 2003. p. 33-43.

19. Silva NS, Nazário CL, Lima FD. Aspectos metodológicos. In: Lebrão ML, Oliveira, YAO, organizadores. SABE - Saúde, Bem-estar e Envelhecimento. O projeto SABE no município de São Paulo: uma abordagem inicial. Brasília: Organização PanAmericana da Saúde; 2003. p. 45-72.

20. Lebrão ML, Duarte YAO. Estudo SABE 2000 e 2006 [CD-ROM]. São Paulo: Universidade de São Paulo; 2008.
21. Lebrão ML, Laurenti R. Saúde, bem-estar e envelhecimento: o estudo SABE no Município de São Paulo. Rev Bras Epidemiol 2005; 8:127-41.

22. Merskey H, Bogduk N. Classification of chronic pain: descriptions of chronic pain syndromes and definitions of pain terms prepared by the International Association for the Study of Pain. 2nd Ed. Seattle: International Association for the Study of Pain; 1994.

23. Lawton MP, Moss M, Fulcomer M, Kleban MH. A research and service multilevel assessment instrument. J Gerontol 1982; 37:91-9.

24. Rao JNK, Scott AJ. The analysis of categorical data from complex sample surveys: chi-squared tests for goodness of fit and independence in two-way tables. J Am Stat Assoc 1981; 76:221-30.

25. Rao JNK, Scott AJ. On chi-squared for multiway contingency tables with cell proportions estimated from survey data. Ann Stat 1984; 12:46-60.

26. Von Korff M, Crane P, Lane M, Miglioretti DL, Simon G, Saunders K, et al. Chronic spinal pain and physical-mental comorbidity in the United States: results from the national comorbidity survey replication. Pain 2005; 113:331-9.

27. Reyes-Gibby CC, Aday L, Cleeland C. Impact of pain on self-rated health in the community-dwelling older adults. Pain 2002; 95:75-82.

28. Scudds RJ, Robertson JM. Pain factors associated with physical disability in a sample of communitydwelling senior citizens. J Gerontol 2000; 55:393-9.

29. Cechi F, Debolini MD, Lova RM, Machhi C, Bandinelli S, Bartali B, et al. Epidemiology of back pain in a representative cohort of Italian persons 65 years of age and older, the In Chianti Study. Spine 2006; 31:1149-55.

30. Di Iorio A, Abate M, Guralnik JM, Bandinelli S, Cecchi F, Cherubini A, et al. From chronic low back pain to disability, a multifactorial mediated pathway: the In Chianti Study. Spine 2007; 32:809-15.

31. Al Snih S, Markides KS, Ray L, Goodwin JS. Impact of pain on disability among older Mexican Americans. J Gerontol A Biol Sci Med Sci 2001; 56:400-4.

32. Odding E, Valkenburg HA, Stam HJ, Hofman A. Determinants of locomotor disability in people aged 55 years and over: the Rotterdam Study. Eur J Epidemiol 2001; 17:1033-41.

33. Turner JA, Ersek M, Kemp C. Self-efficacy for managing pain is associated with disability, depression, and pain coping among retirement community residents with chronic pain. J Pain 2005; 6:471-9.

34. Menz HB, Morris ME. Determinants of disabling foot pain in retirement village residents. J Am Podiatr Med Assoc 2005; 95:573-9.

35. Gilmour H, Park J. Dependency, chronic conditions and pain in seniors. Health Rep 2006; 16 Suppl:21-31.

36. Miró J, Paredes S, Rull M, Queral R, Miralles R, Nieto $\mathrm{R}$, et al. Pain in older adults: a prevalence study in the Mediterranean region of Catalonia. Eur J Pain 2007; 11:83-92.

37. Rudy TE, Weiner DK, Lieber SJ, Slaboda J, Boston RJ. The impact of chronic low back pain on older adults: a comparative study of patients and controls. Pain 2007; 131:293-301. 
38. Covinsky KE, Lindquist K, Dunlop DD, Yelin E. Pain, function limitations, and aging. J Am Geriatr Soc 2009; 57:1556-61.

39. Eggermont LH, Bean JF, Guralnik JM, Leveille SG. Comparing pain severity versus pain location in the MOBILIZE Boston study: chronic pain and lower extremity function. J Gerontol A Biol Sci Med Sci 2009; 64:763-70.
40. Weiner DK, Rudy TE, Kim YS, Golla S. Do medical factors predict disability in older adults with persistent low back pain? Pain 2004; 112:214-20.

Recebido em 04/Mai/2012

Versão final reapresentada em 22/Set/2012 Aprovado em 03/Out/2012 